\title{
Thirst in patients admitted to intensive care units: an observational study
}

\author{
Alessandra Negro ${ }^{1}$ - Giulia Villa ${ }^{2}$ - Massimiliano Greco ${ }^{3}$ - Eleonora Ciriolo ${ }^{1}$ - Elisabetta Livia Luraschi ${ }^{1}$. \\ Jacopo Scaramuzzi ${ }^{1} \cdot$ Duilio Fiorenzo Manara ${ }^{2} \cdot$ Alberto Zangrillo $^{4}$
}

Received: 10 August 2020 / Accepted: 13 October 2021 / Published online: 19 October 2021

(c) The Author(s), under exclusive licence to Royal Academy of Medicine in Ireland 2021

\begin{abstract}
Background Despite various studies reporting a high prevalence, reaching $71 \%$, the sensation of thirst in intensive care unit (ICU) patients, its prevention, detection, and management, is not well known nor considered. Limited research has examined the causes of thirst in ICU patients, while it has been examined in other patient populations.

Aim To determine the incidence and intensity of thirst in patients admitted to ICU and its association with airway devices (endotracheal tube, tracheostomy, oxygen mask), airway humidification, patients' characteristics, and therapy (serum sodium concentration, hematocrit, fluid balance, possibility of oral hydration, and dosage of diuretics).

Methods Patients were interviewed daily to report the presence of thirst and rate its intensity on a Numeric Rating Scale (NRS) from 0 (no thirst) -10 (intolerable thirst). Other data were obtained through direct evaluation or by consulting medical records. Patients admitted to three ICUs from May to August 2014 in a university hospital in Italy were included.

Results A total of 220 Patients were enrolled. Thirst was found in $76.1 \%$ of patients' observations, with a mean thirst score of 5.37. Thirst intensity was predicted by high doses of diuretics (>100 mg/die), increasing serum sodium concentration, absence of oral hydration and the presence of xerostomia. Thirst was associated with the use of humidified Venturi mask.

Conclusions Thirst is highly prevalent among patients in this population of intensive care patients. It would be desirable to evaluate this stressor at least daily, to eliminate or relieve this sensation.
\end{abstract}

Keywords Critically ill patient · Thirst

\section{Introduction}

Thirst is an important physiological stimulus to maintain body fluid homeostasis. There are many regulatory mechanisms that preserve a constant body water volume, including thirst, as one of the main system for human survival [1,2]. Over two decades ago, Porth and Erickson defined thirst as a subjective feeling, closely related to a strong desire to

Giulia Villa

villa.giulia@hsr.it

1 IRCCS San Raffaele Institute, Milan, Italy

2 Center for Nursing Research and Innovation, Vita-Salute San Raffaele University, Via Olgettina, 58, 20132 Milan, Italy

3 IRCCS Humanitas Clinical and Research Center, Milan, Italy

4 IRCCS San Raffaele Institute, Vita-Salute University, Milan, Italy drink, usually expressed as an urge to ingest fluids or foods with a high-water content. These authors identified thirst as a clinical issue in the intensive care unit (ICU) underlying that it frequently occurs in patients with hypovolemia, or heart or kidney failure, especially in the ICU despite intravenous hydration, due to patients' inability to drink fluids [3]. Moreover, there are specific conditions in ICU patients, which increase water demand. Reasons include xerostomia (dry mouth), mouth breathing, administration of dry medical gases, the presence of tracheal tubes, patient inability to drink or to communicate desire to drink, or treatments with medications such as anticholinergic, diuretics, sympathomimetic, and antihypertensive agents [4]. Thirst could be avoided or at least reduced if routinely assessed with specific instruments that assess the intensity of thirst, hydration of the mucosa, and dry mouth [5].

In a study, Chanques et al. [6] stated that thirst is one of the five ICU patient symptoms that should be evaluated daily, because it is the most prevalent and intense symptom 
reported by ICU patients, but assessment alone does not reduce or avoid thirst, intervention is required. Some experience emerged aimed at identifying strategies to alleviate thirst in ICU patients [5, 7-11]. Identification of patients at risk and early intervention should be standard practice within ICUs $[8,9]$. The relevance of the topic does not emerge in medical and nursing literature, but the scientific debate needs to focus on thirst in ICU patients: in fact, while thirst is one of the most intensely perceived symptoms, the first $[12,13]$ or the second [14] most prevalent symptom in ICUs, there is a lack in ICU nursing research regarding its assessment, correlation to patient deterioration, or to agitation state, diagnosis, and interventions. This is remarkable, as thirst is primarily identified by nurses within hospitals [3]. Another relevant point is mouth care in ICU patients that could better alleviate the sensation of thirst, but gaps in practice and scarce researchers' attention, except for ventilator-associated pneumonia (VAP) prevention, make this intervention highly variable.

\section{Thirst in ICU patients: prevalence and intensity}

In a University ICU study of Li and Puntillo, researchers focused their attention on thirst in ICU patients: it was reported as severe by $40 \%$ and moderate by $30 \%$ of patients, with an average intensity of 5.7 on a 0 (no thirst) to 10 (intolerable thirst) scale [15]. According to an interesting literature review, in the ICU settings, thirst is reported as the highest source of stress, with a prevalence in ICU patients of $70.8 \%$ [16]. Kalfon et al. [17] found that thirst was a stressor in $60 \%$ of patients, with $17.9 \%$ patients reporting a thirst intensity over 70 , on a $0-100$ scale. Thirst was associated with xerostomia, endotracheal tubes, tracheostomies, oxygen therapy, solid and liquid fasting, electrolyte alterations, and hypovolemia. In a recent single-blind randomized trial conduced in three ICUs in a tertiary medical center [18], oral swab wipes, sterile ice-cold water sprays, and lip moisturizer significantly reduced thirst intensity and distress. Stotts et al. [16, 19] found that doses of opioids $>50 \mathrm{mg}$, or furosemide $>60 \mathrm{mg}$, or selective serotonin re-uptake inhibitors were associated with thirst, whereas no oral hydration and gastrointestinal disorders increased thirst intensity. Distress, mechanical ventilation, negative fluid balance, and the use of antihypertensive drugs had similar outcomes.

\section{Thirst and ICU nurses}

Landstrom and colleagues [20] in 2009 interviewed ICU nurses about thirst. ICUs nurses are uniquely placed to assess and treat patients' thirst; however, there are gaps in nursing knowledge of thirst prevalence and consequence in ICU. Half the number of respondents did not perceive thirst as a problem for patients, and thirst is not commonly mentioned during handovers or in nursing documentation.

No study has yet considered to adapt nursing care to patients' thirst, even when thirst was experienced.

Our purpose is to assess thirst incidence in ICU patients and its associated risk fact.

\section{Aim of the study}

This prospective, observational study was performed to investigate the incidence of thirst in ICU patients, and to assess association of thirst with endotracheal tube, tracheostomy, spontaneous breathing, oxygen therapy with or without humidification, dry mouth, mouth breathing, and condition of the oral mucosa.

\section{Methods}

\section{Study design}

The study design is a prospective observational study.

\section{Population and setting}

From May 1, 2014 to August 31, 2014, patients admitted to the general ICU, ( 8 beds, 500 patients/year, including trauma, septic, and respiratory patients), cardiac surgery ICU (14 beds, 1200 patients/year), and neurosurgery ICU ( 6 beds, 150 patients/year) of a large University Hospital in Italy were included in the study. Nurse:patients' ratio was $1: 2$ in all the ICU. Inclusion criteria were age $\geq 18$ years, Glasgow Coma Scale $\geq 9$, spontaneously breathing, and with tracheal intubation or tracheostomy at recruitment. Patients who were confused could be potentially included and those unable to speak were aided letting them write on a white board. Patients who did not speak Italian language were excluded.

\section{Data collection}

Nurses interviewed patients once daily, between $6.00 \mathrm{a} . \mathrm{m}$. and 2.00 p.m., the period of the day that allows collecting the most robust and replicable data, for the entire length of ICU stay. Thirst was investigated by asking patients to quantify the intensity at that moment on a Numeric Rating Scale from 0 (no thirst) to 10 (intolerable thirst) (NRS). Patients who could not respond verbally answered questions by writing on a white board. Training was performed 
by the principal investigators to the staff, to limit variability on data collection.

For each patient, we collected sex, age, reason for admission and admitting ICU, ICU length of stay, serum sodium, hematocrit, diuretic therapy, oral hydration or use of gauze to moisten lips, timing from last oral hygiene, presence of xerostomia, and thirst on a Numeric Rating Scale from 0 to 10 [16]. Additionally, we registered the presence of endotracheal or tracheostomy tube, spontaneous breathing, type and humidification of oxygen mask, mouth breathing, and status of the oral mucosa.

Diuretic therapy with furosemide was arbitrarily divided in low dose (less than $100 \mathrm{mg} /$ day), and high dose (over $100 \mathrm{mg} /$ day). Furosemide is the most common diuretic prescribed for patients; other diuretic drugs are sporadically used and were not considered in this study.

\section{Data analysis}

Data were analyzed using Stata (StataCorp. 2015. Stata Statistical Software: Release 14. College Station, TX: StataCorp LP). Continuous data are reported as mean (standard deviation) or median, (interquartile range) as appropriate. Categorical data are reported as frequency (percentage). A first level of exploratory analysis was conducted using chi square and Kruskal-Wallis tests, without considering panel data. We performed univariate analysis using mixed effect, considering panel data.

We built a multilevel-ordered logistic regression model, using post-estimation likelihood ratio test (LR test) for inclusion of new variables in the model. We decided to test a priori the interaction between the use of wet gauzes and xerostomia, and between oral hydration and xerostomia, as these are simple and effective procedure prompted by nurses that may have a multiplicative effect on thirst level. We categorized thirst in a three ordinal level variable (no/low thirst $=$ NRS $0-3$, moderate thirst $=4-7$, severe thirst $=8-10$ ). Differences at $p$ values $<0.05$ were considered significant. The proportional odds assumption was tested by Brant test of parallel regression.

\section{Ethics}

The study was approved by the Institutional Review Board. Eligible patients (or their relatives in case of patient's inability) signed written consent before inclusion and relevant clearance obtained. Data were collected anonymously, on a predefined paper form and then inserted into an electronic database.
Table 1 Reasons for admissions of the patients in the ICUs

\begin{tabular}{llll}
\hline Reasons for admission & \multicolumn{2}{l}{ Number of patients } \\
\cline { 2 - 4 } & $\begin{array}{l}\text { General } \\
\text { ICU }\end{array}$ & $\begin{array}{l}\text { Cardiac } \\
\text { surgery } \\
\text { ICU }\end{array}$ & $\begin{array}{l}\text { Neurosurgery } \\
\text { ICU }\end{array}$ \\
\hline Post surgery monitoring & 18 & 142 & 6 \\
Septic shock & 5 & 1 & 2 \\
Respiratory failure & 8 & 1 & 2 \\
Cardiac arrest and other & 3 & 16 & 0 \\
$\quad$ cardiac problems & & & \\
Trauma & 2 & 0 & 1 \\
Toxic problems & 2 & 0 & 0 \\
Neurological diseases & 1 & 0 & 5 \\
Other & 2 & 3 & 0 \\
Total & 41 & 163 & 16 \\
\hline
\end{tabular}

\section{Results}

A total of 268 out of 607 (36.4\%) patients fulfilled inclusion criteria, with 220 signing written consent and being included in the study, 72,4 of them were male (160). There were 41/71 (59\%) patients from general ICU, 163/426 (38\%) from cardiac ICU, and 16/110 (15\%) from the neurosurgical ICU. Demographic information is shown in Tables 1 and 2. The ICU length of stay ranged from 1 to 117 days, with a mean of 5.90 (SD 13.30) days.

A total of 707 thirst observations were recorded, yielding a mean thirst NRS of $5.37(\mathrm{SD} \pm 3.80)$. Thirst did not differ according to the ICU, with a $5.49(\mathrm{SD} \pm 3.71$, 234 observations) mean score in the general ICU, 5.43 ( $\mathrm{SD} \pm 3.92,37$ observations) in the cardiac ICU, and 4.89 ( $\mathrm{SD} \pm 3.63,103$ observations) in the Neurosurgical ICU $(p=0.33)$. A NRS equal to 10 was present in $23.90 \%$ (169 observations) and was less frequent in the neurosurgical ICU (15.50\%). A total of 277 observations (26.50\%) reported a NRS $\geq 8$, while 445 observations $(52.20 \%)$ had a NRS $\geq 5$. No thirst (NRS $=0$ ) was detected only in 170 $(24.10 \%)$ observations. When considering mean patient thirst, age and gender were not significantly different between male (NRS 5.75 \pm 3 ) and female (NRS 5.25 \pm 3 ) $(p=0.4)$, nor between younger (NRS $6 \pm 3.2)$ vs older (NRS 5.5 \pm 3.1$)$ patients $((p=0.4)$,

Table 2 Patient age according to enrolling ICU

\begin{tabular}{llll}
\hline Age & Mean & $\begin{array}{l}\text { Standard } \\
\text { deviation }\end{array}$ & Range \\
\hline General ICU & 65 & 43.84 & $22-84$ \\
Cardiac ICU & 61 & 46.66 & $19-85$ \\
Neurosurgery ICU & 53 & 37.47 & $21-74$ \\
Total & 61 & 46.66 & $19-85$ \\
\hline
\end{tabular}




\section{Exploratory univariate analysis not accounting for panel data}

High-dose diuretics were associated with higher thirst NRS (6.3 [sd 3.7] vs 4.8 [sd 3.9], $p<0.001$ ). However, low-dose diuretics did not influence thirst NRS when compared to patients without diuretic therapy (4.8 [3.9] vs 5.2 [3.7], $p=0.27$ ). Oral hydration was associated with a reduction in thirst, with a mean thirst score of $5.0 \pm 3.6$ for patients receiving oral hydration vs $5.8 \pm 3.9$ for patients without oral hydration $(p<0.01)$. Serum sodium levels over $140 \mathrm{mmol} / 1$ were poorly associated with the highest NRS values $(p<0.01, r=0.10)$. The hematocrit level was not associated with thirst $(p=0.4, r=0.03)$. Time elapsed from the last oral hygiene showed a trend toward higher NRS for increasing time (NRS $5 \pm 3.8$ for lower class vs $9 \pm 1$ for higher class), with poor correlation significance ( $p=0.4$, $r=0.02) 4)$. Xerostomia was associated with increased thirst (median NRS $7-3 \pm 3$ vs $4.7 \pm 3.8,(p<0.001)$ (Fig. 1$)$; this was confirmed even when considering data from the 3 ICUs separately.

Thirst scores differed according to the type of breathing device employed. Patients with nasal cannulae reported lower thirst scores than those with humidified Venturi masks $(4.7 \pm 3-6$ vs $5.5 \pm 3.6, p=0.04)$ and non-humidified Venturi masks $(4.7 \pm 3.6$ vs $6-3 \pm 3.9, p<0.01)$ (Fig. 2).

\section{Ordered logistic regression with mixed effect}

A multilevel mixed effect-ordered logistic regression model was built. When controlling for the other factors, thirst levels are increased by high-dose diuretics (OR 2.25 [95\% CI 1.38-3.65] $p=0.001$ ) for severe thirst versus combined lower levels, and for combined higher levels versus low thirst, while low dose of diuretics had no effect on thirst level. For each increase in $5 \mathrm{mmol} / \mathrm{L}$ of serum sodium level, there was a $22 \%$ increase $(95 \%$ CI $7-39 \%, p=0.003)$ in proportional odds for severe thirst versus combined lower levels, and for combined higher levels versus low thirst. The use of wet gauze is associated with increased thirst (OR 1.81 [95\%CI 1.03-3.16], $p=0.038$ ). The effect of xerostomia on

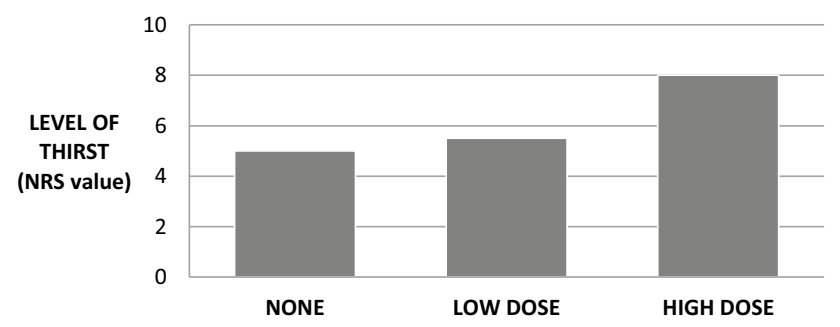

Fig. 1 Association of perceived thirst and diuretic dose (none; low diuretic dose: $<100 \mathrm{mg} /$ day, high diuretic dose: $>100 \mathrm{mg} /$ day)

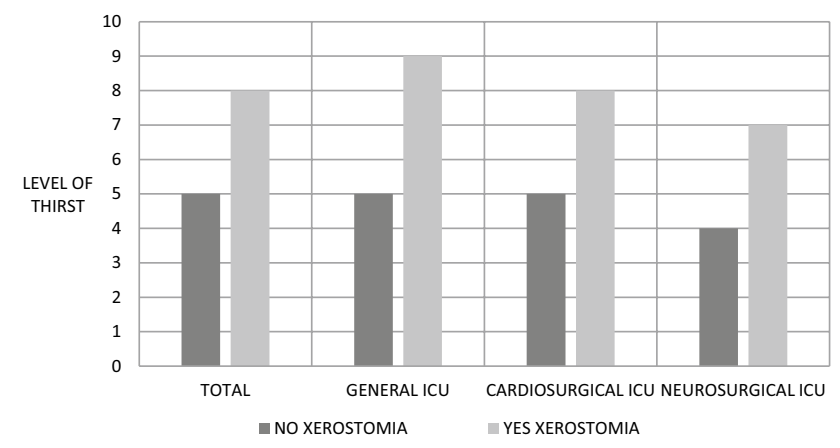

Fig. 2 Association between median thirst level and xerostomia

thirst level depends on the use of wet gauzes, with a significant interaction between the two ( $p$ for interaction $<0.001$ ). Xerostomia affected proportional odds for thirst by a 6.55 fold increase (95\% CI 3.56-12.03, $p<0.001)$. Using wet gauzes in patients with xerostomia reduced, the combined effect to a 5.14-fold increase in proportional OR $(95 \% \mathrm{CI}$ $2.23-11.85 \%, p<0.001)$, albeit the effect due to wet gauzes was not significant $(p=0.5)$. Oral hydration did not influence the level of thirst in these observed patients (OR 1.24 [0.69-2.21]). Considering airway/oxygen delivery devices maintaining nasal cannula as baseline level, there was no difference for tracheostomy, tracheal intubation, standard Venturi mask, or noninvasive ventilation. Only Venturi masks with active heat humidification were associated with increased odds for thirst (proportional OR 1.94 [95\% CI 1.31-2.87], $p=0.001$ ).

The proportional odds assumption was tested by Brant test of parallel regression $(p=0.16)$.

When considering only unaided, spontaneously breathing patients in the multilevel-ordered logistic regression model, there was a significant effect on thirst exerted xerostomia (OR 5.9 [1.3-26.9], $p=0.02$ ), oral hydration (OR 3.5 [1.3--9.7]), use of wet gauzes (3.4 [1.3-9.2]), low (OR 3.7 [1.3.10.3]) or high (OR 6.3 [1.7-22.9] diuretic dose.

\section{Univariate analysis accounting for panel data}

When mixed effect logistic regression was employed, considering panel data in univariate analysis, high-dose diuretics were associated with thirst when compared to no diuretic therapy (OR 2.2 (95\% CI 1.4-3.6], $p<0.001$ ). No association was found for low-dose diuretics (OR 1.2, 95\% CI $0.8-1.8$ ), age (OR for every 10 years $1.1,95 \%$ CI $0.98-1.3$ ) or male gender (95\% CI 0.94-2.0).

Xerostomia was significantly associated with thirst (OR $4.5,95 \%$ CI 2.9-6.9, $p<0.001$. Increasing sodium level was significantly associated with thirst (OR 1.3, 95\% CI 1.1-1.4, $p<0.001)$. 
The effects of different airway device compared to baseline (nasal cannula) are reported in Table 3.

\section{Discussion}

The purpose of the study was to assess thirst incidence in ICU patients and its associated risk factors. Results show that thirst is a frequent and intense sensation in this population of critically ill patients. A total of 537 of observations $(76.1 \%)$ reported thirst, with a mean score of 5.4/10.

When considering all risk factors for thirst, thirst was associated with the following: (1) absence of oral hydration, (2) the use of wet gauzes, (3) the presence of xerostomia, which depended also on the use of wet gauzes, and (4) high diuretic dose. Among respiratory devices, only humidified Venturi mask was associated with thirst level.

These results are similar with those of Puntillo's work [16], in which thirst was detected in $70.8 \%$ of the assessment, with a mean intensity of 2.16 , even if using a NRS from one to three $(1=$ mild, $2=$ moderate, $3=$ severe $)$. Arai et al. [14] presented similar results, with $70 \%$ of patients reporting moderate to severe thirst on a NRS from 4 to 10 . As several studies often adopt different scales, there is need for common criteria and scales to define and detect thirst to make studies comparable.

$\mathrm{Li}$ and Puntillo [15] evaluated the intensity of some of the main symptoms perceived in the ICU, including thirst in patients undergoing mechanical ventilation. They used a 0-10 NRS and enrolled 15 ICU patients with a mean ICU stay of 12 days. The study showed that $40 \%$ of patients reported severe thirst (7-10), 33\% of patients moderate thirst (4-6), $6 \%$ of patient mild thirst (1-3), and $20 \%$ of patients no thirst (0). These results can be compared to the 26 patients on mechanical ventilation from our study (159 observations). We found severe thirst (7-10) in $41 \%$ of observations, moderate thirst in $19 \%$ of observations, mild thirst in $7.5 \%$ of observations, and no thirst in $32 \%$ of observations. Differences may be due to the relatively small sample sizes in both studies. Two other studies were carried out in general, cardiac surgery and neurosurgery ICUs, and had sample size comparable with our study. They assessed thirst by a $0-10$

Table 3 The effect of oral devices on thirst, compared with baseline (nasal cannula)

\begin{tabular}{lll}
\hline & OR $(95 \% \mathrm{CI})$ & $p$ \\
\hline Orotracheal intubation & $3.8(1.2-12.1)$ & 0.019 \\
Venturi mask & $1.9(0.99-3.7)$ & 0.05 \\
Humidified Venturi mask & $1.7(1.1-2.4)$ & 0.007 \\
Tracheostomy & $1.6(1.1-2.4)$ & 0.01 \\
NIV & $0.9(0.4-1.8)$ & 0.6 \\
\hline
\end{tabular}

NRS, including only patients with an NRS $\geq 3$. Stotts et al. reported a mean thirst score of 6.67 [19], while Puntillo et al. reported a mean NRS between 5 and 6 in 252 patients [18]. In our study, when considering only NRS between 3 and 10 , the mean score was 7.4 . Kalfon et al. investigated the sensation of thirst and its possible intensity on a VAS from 0 to 100 , and found $17.9 \%$ of patients reporting VAS $\geq 70$ [17]. Our results show a higher incidence of severe thirst, with $44.9 \%$ of observations reporting NRS $\geq 7$. Similarly, the former study yielded a $32 / 100(\mathrm{SD} \pm 34)$ mean thirst score, which can be compared to the mean NRS of 5.37/10 in our study. This variability can be due to differences in data collection, as Kalfon et al. collected data retrospectively at ICU discharge, while we collected data prospectively on a day-to-day basis. Moreover, in the former study, women experienced greater thirst (39 vs 28 ) than men, and surgical patients' greater thirst than medical patients (37 vs 25 ), while these results were not confirmed in our study. Elderly patients perceive thirst with an intensity lower than that of younger people, therefore increasing their risk of dehydration [14]. Data in this study did not find a significant association between age and thirst level. Shikha et al. systematically review the literature about intervention. They found that intervention bundle consisting of more than one intervention is significantly effective in reducing thirst and dry in ICU patients [10]. Zhang et al. performed a RCT in order to test an intervention bundle (vitamin $\mathrm{C}$ sprays, peppermint water mouthwash, and a lip moisturizes) to relieve thirst and dry mouth. A total of 61 patients were recruited to the study. The average decrease in thirst intensity and oral mucosa situation scores after the interventions was larger in the experimental group patients relative to controls ( 1.27 and 0.36 vs. 0.19 and 0.1 points, respectively; $p<0.05$ ). Being male, not receiving diuretics, and having higher serum sodium levels were potential predictors of thirst and oral dryness [5].

Several studies have highlighted the association between levels of serum sodium and thirst intensity [2, 5, 14]. Data in this study showed, when controlling for other factors, serum sodium were significantly associated with thirst intensity (22\% increase in OR [7-39\%]) for a $5 \mathrm{mmol} / \mathrm{L}$ increase $p=0.003$ ). This confirms the findings by Arora on the effects of natremia on plasma osmolality and consequently on thirst [2]. Furthermore, up to $70 \%$ of the stimuli leading to thirst were caused by imbalances in plasma osmolality in Arai et al. mainly driven by sodium values greater than $145 \mathrm{mmol} / \mathrm{l}[14]$. The retrospective study by Stelfox reported hypernatremia ( $\geq 145 \mathrm{mmol} / \mathrm{l}$ ) in about $26 \%$ of patients in ICU, while our study found hypernatremia in $10 \%$ of patients [21].

Other factors that have been previously reported to be associated with thirst were xerostomia, and the use of wet gauzes. Thelin et al. in 2008 concluded that some situations such as chronic diseases, surgeries, and aging affect 
the production of the salivary glands, leading to xerostomia [22]. The absence of an adequate salivation in patients with orotracheal intubation could contribute to the development of mucositis and colonization by gram-negative bacteria [4]. Chanques et al. concluded that in spontaneously breathing ICU patients undergoing high-flow oxygen therapy, the use of heated humidifiers significantly decreases xerostomia [23]. Our study did not confirm this finding. In our data, heated humidified Venturi masks were associated with thirst: this result may reflect nurses' and physicians' beliefs on the positive effect of heated humidification, leading heated humidified Venturi mask to be more frequently employed in patients complaining of thirst. In the observed setting in fact there is no unique indication to use heated/humidified oxygen delivery for all patients.

Arai et al. underline the presence of receptors in the oropharyngeal region, playing an important role in thirst regulation [24]. This is the rationale behind the use of wet gauzes, a procedure commonly and autonomously employed by nurses. In our study, the use of wet gauze with water, without any specific oral care local guidelines in use across the studied services, reduced thirst scores in patients with xerostomia. Thus, wet gauzing to humidify lips and tongue may be a simple and appropriate strategy to reduce thirst in all patients. In accordance with published studies, we did not find any association between gender and thirst, neither in univariate or multivariate analysis. We conclude that gender is not a risk factor for thirst in ICU patients.

Thirst should be systematically assessed daily in ICU patients $[5,8,10]$. Assessors should evaluate also its described risk factors, to detect thirst also in unconscious patients and in patients temporarily unable to communicate their sensations, such as those affected by delirium. Moreover Sato et al. recently found that thirst persisting more than $24 \mathrm{~h}$ was significantly associated with increased risk for delirium [25].

\section{Limits}

This is a single center study, so results could lack external validity. Oral hydration was recorded without considering the cumulative water intake provided to patients. Oral mucosa was evaluated clinically, but this method is prone to subjective evaluation even when employed by trained personnel. There is need for standardization in future studies, including the development of instrument to objectively evaluate the conditions of the oral cavity. In this study, data were collected daily between 6:00 a.m. to 2:00 p.m., and as oral hygiene was conducted in morning shift, there are no data over $5 \mathrm{~h}$ after oral hygiene. This may hinder the effect of oral hygiene on thirst that may be more and more evident as time from this procedure increases. While we used a 0-10 NRS to be consistent with published literature, the most recorded values were at extreme ends of the scale ( 0 and 10$)$, and this may decrease the precision of this scale. Clinicians' attitude on thirst assessment should also be evaluated in further studies, including strategies to increase their level of awareness on thirst and related consequences.

\section{Conclusion}

The incidence of thirst among ICU patients is high (76.1\% of observations, median NRS 5.3/10). Thirst is associated with the use of high-dose diuretics, no oral hydration, and xerostomia. Breathing devices did not influence thirst, when controlling for other factors. Despite its high prevalence, thirst and correlated elements are not well known nor considered in daily practice. It is advisable to evaluate thirst and related factors once daily at minimum, with the aim of identifying means of alleviating this discomforting condition. In addition, the use of intervention bundles in a systematic way can be a valuable tool to support clinical practice in combating thirst.

\section{Impact statement}

What does this paper contribute to the wider global clinical community?

- While thirst is one of the most intensely perceived symptoms, the second most prevalent symptom in ICUs [13], there is a lack in nursing research regarding its assessment, diagnosis, and interventions.

- There is a high prevalence of thirst among ICU patients (76.1\% of subjects, median 5.37).

- Thirst was associated with the use of high doses of diuretics, absence of oral hydration, and presence of xerostomia. Humidified Venturi masks were the only breathing devices associated with thirst.

- Thirst should be systematically assessed in ICU patients. Assessors should evaluate also its described risk factors, to detect thirst also in unconscious patients and in patients temporarily unable to communicate their sensations, such as those affected by delirium.

Author contribution Conceptualization: AN, GV. Methodology: AN, MG, GV. Formal analysis and investigation: AN, GV, MG, EC, EL, JS. Writing - original draft preparation: AN, MG, GV. Writing - review and editing: AN, GV. Supervision: DFM, AZ. 
Data availability All data and materials claim and comply with the standards.

Code availability All statistical analyses were performed with the STATA software (ver. 16; Texas USA).

\section{Declarations}

Ethics approval The study was approved by the Ethical Committee.

Consent to participate and consent for publication Consent to participate in the study and for publication was obtained from all participants included in the study.

Conflict of interest The authors declare no competing interests.

\section{References}

1. Adams J, Myatich AI, McCullough AS (2020) Thirst as an ingestive behavior: a brief review on physiology and assessment. Nutr Health 26(3):271-274. https://doi.org/10.1177/0260106020916972

2. Arora SK (2013) Hypernatremic disorders in the intensive care unit. J Intensive Care Med 28(1):37-45. https://doi.org/10.1177/ 0885066611403994

3. Porth CM, Erickson M (1992) Physiology of thirst and drinking: implication for nursing practice. Heart Lung 21(3):273-282

4. Dennesen $\mathrm{P}$ et al (2003) Inadequate salivary flow and poor oral mucosal status in intubated intensive care unit patients. Crit Care Med 31(3):781-786. https://doi.org/10.1097/01.CCM. 0000053646.04085 .29

5. Zhang W, Gu Q, Gu Y, Zhao Y, Zhu L (2021) Symptom management to alleviate thirst and dry mouth in critically ill patients: a randomised controlled trial. Aust Crit Care. https://doi.org/10. 1016/j.aucc.2021.04.002

6. Chanques G, Nelson J, Puntillo K (2015) Five patient symptoms that you should evaluate every day. Intensive Care Med 41(7):1347-1350. https://doi.org/10.1007/s00134-015-3729-x

7. Puntillo K, Arai SR, Cooper BA, Stotts NA, Nelson JE (2014) A randomized clinical trial of an intervention to relieve thirst and dry mouth in intensive care unit patients. Intensive Care Med 40(9):1295-1302. https://doi.org/10.1007/s00134-014-3339-z

8. Leemhuis A, Shichishima Y, Puntillo K (2019) Palliation of thirst in intensive care unit patients: translating research into practice. Crit Care Nurse 39(5):21-28. https://doi.org/10.4037/ccn2019544

9. Adams JD, Darcy C, DeGrasse AG, Jordan R, Boscia CS (2021) "Crosstalk of pain and thirst perception: a brief review". Chemosens Percept 1-4. https://doi.org/10.1007/s12078-021-09286-3

10. Shikha G, Vinay K, Neetu K (2020) Effectiveness of an intervention bundle on thirst intensity and dry mouth among patients admitted in intensive care units. Indian J Public Heal Res Dev 11:101-106

11. VonStein M, Buchko BL, Millen C, Lampo D, Bell T, Woods AB (2019) "Effect of a scheduled nurse intervention on thirst and dry mouth in intensive care patients". Am J Crit care an Off Publ Am. Asso Crit Nurses 28(1):41-46. https://doi.org/10.4037/ ajcc2019400

12. Gultekin Y, Ozcelik Z, Akinci SB, Yorganci HK (2018) Evaluation of stressors in intensive care units. Turkish J Surg. https://doi. org/10.5152/turkjsurg.2017.3736

13. Zengin N, Ören B, Üstündag H (2020) The relationship between stressors and intensive care unit experiences. Nurs Crit Care 25(2):109-116. https://doi.org/10.1111/nicc.12465

14. Arai S, Stotts N, Puntillo K (2013) Thirst in critically ill patients: from physiology to sensation. Am J Crit Care 22(4):328-335. https://doi.org/10.4037/ajcc2013533

15. Li DT, Puntillo K (2006) A pilot study on coexisting symptoms in intensive care patients. Appl Nurs Res 19(4):216-219. https:// doi.org/10.1016/j.apnr.2006.01.003

16. Puntillo KA et al (2010) Symptoms experienced by intensive care unit patients at high risk of dying. Crit Care Med 38(11):21552160. https://doi.org/10.1097/CCM.0b013e3181f267ee

17. Kalfon $\mathrm{P}$ et al (2010) Development and validation of a questionnaire for quantitative assessment of perceived discomforts in critically ill patients. Intensive Care Med 36(10):1751-1758. https:// doi.org/10.1007/s00134-010-1902-9

18. Puntillo K et al (2014) Palliative care in the ICU: relief of pain, dyspnea, and thirst - a report from the IPAL-ICU Advisory Board. Intensive Care Med 40(2):235-248. https://doi.org/10.1007/ s00134-013-3153-z

19. Stotts NA, Arai SR, Cooper BA, Nelson JE, Puntillo KA (2015) Predictors of thirst in intensive care unit patients. J Pain Symptom Manage 49(3):530-538. https://doi.org/10.1016/j.jpainsymman. 2014.07.001

20. Landström M, Rehn I-M, Frisman GH (2009) Perceptions of registered and enrolled nurses on thirst in mechanically ventilated adult patients in intensive care units - a phenomenographic study. Intensive Crit Care Nurs 25(3):133-139. https://doi.org/10.1016/j. iccn.2009.03.001

21. Stelfox H, Ahmed SB, Khandwala F, Zygun D, Shahpori R, Laupland $\mathrm{K}$ (2008) The epidemiology of intensive care unit-acquired hyponatraemia and hypernatraemia in medical-surgical intensive care units. Crit Care 12(6):R162. https://doi.org/10.1186/cc7162

22. Thelin $\mathrm{W}$ et al (2008) The oral mucosa as a therapeutic target for xerostomia. Oral Dis 14(8):683-689. https://doi.org/10.1111/j. 1601-0825.2008.01486.x

23. Chanques $\mathrm{G}$ et al (2009) Discomfort associated with underhumidified high-flow oxygen therapy in critically ill patients. Intensive Care Med 35(6):996-1003. https://doi.org/10.1007/ s00134-009-1456-x

24. Arai SR, Butzlaff A, Stotts NA, Puntillo KA (2014) Quench the thirst. Biol Res Nurs 16(4):456-466. https://doi.org/10.1177/ 1099800413505900

25. Sato K, Okajima M, Taniguchi T (2019) Association of persistent intense thirst with delirium among critically ill patients: a cross-sectional study. J Pain Symptom Manage 57(6):1114-1120. https://doi.org/10.1016/j.jpainsymman.2019.02.022

Publisher's Note Springer Nature remains neutral with regard to jurisdictional claims in published maps and institutional affiliations. 